\title{
Adesão ao tratamento anti-hipertensivo e ocorrência de Síndrome Metabólica
}

\author{
Adherence to antihypertensive treatment and occurrence of Metabolic Syndrome \\ Adherencia al tratamiento antihipertensivo y la ocurrencia del Síndrome Metabólico
}

\begin{abstract}
Geiciane Fonteles da Silva ${ }^{1}$ (C)
Paulo Sávio Fontenele Magalhães ${ }^{2}$ (1)

Vagner Rodrigues Silva Junior ${ }^{3}$ (1)

Thereza Maria Magalhães Moreira ${ }^{1,2,3}$ (D)

1. Universidade Estadual do Ceará. Fortaleza, $\mathrm{CE}$, Brasil.

2. Universidade Estadual do Ceará, Programa de Pós-graduação em Saúde Coletiva.

Fortaleza, CE, Brasil.

3. Universidade Estadual do Ceará, Programa de Pós-graduação em Cuidados Clínicos em Enfermagem e Saúde. Fortaleza, CE, Brasil.
\end{abstract}

Autor correspondente:

Vagner Rodrigues Silva Junior.

E-mail: vagnerrsjunior@yahoo.com.br

Recebido em 09/06/2020.

Aprovado em 21/09/2020.

DOl:https://doi.org/10.1590/2177-9465-EAN-2020-0213

\section{RESUMO}

Objetivo: Analisar a associação entre a adesão ao tratamento anti-hipertensivo e a ocorrência de Síndrome Metabólica em pacientes hipertensos de uma unidade de atenção primária à saúde. Método: Estudo analítico com corte transversal realizado com 306 pacientes hipertensos de uma Unidade de Atenção Primária à Saúde localizada na cidade de Fortaleza-CE. Resultados: Dos participantes do estudo $74,2 \%$ eram do sexo feminino, média $61,8 \pm 11,9$ anos e dentro da faixa etária de 54 a $69,77,1 \%$ raça/cor parda, 60,8\% com nível fundamental incompleto ou completo e 70,6\% com renda familiar entre 1 e 2 salários mínimos. Dos hipertensos avaliados, $41,5 \%$ apresentaram Síndrome Metabólica e 76,5\% apresentava aderência parcial ao tratamento anti-hipertensivo. Na avaliação estatística da associação entre as variáveis foi verificado não existir ( $p=0,149$ e $p=0,642)$. Os valores referentes à adesão ao tratamento anti-hipertensivo eram iguais, tanto nos pacientes com Síndrome Metabólica como dos que não apresentavam essa condição. Conclusão e implicações para a prática: A adesão terapêutica anti-hipertensiva alta e parcial foi prevalente. A frequência de Síndrome Metabólica entre os hipertensos em estudo foi elevada. Portanto, medidas de prevenção para doenças cardiovasculares e alcance das metas terapêuticas devem ser implementas continuamente. Um desafio a ser superado pelos profissionais e serviços de saúde.

Palavras-chaves: Cooperação e Adesão ao Tratamento; Hipertensão; Síndrome Metabólica; Medidas de Associação, Exposição, Risco ou Desfecho; Atenção Primária à Saúde.

\section{ABSTRACT}

Objective: To analyze the association between adherence to antihypertensive treatment and the occurrence of Metabolic Syndrome in hypertensive patients in a primary health care unit. Method: Analytical cross-sectional study carried out with 306 hypertensive patients from a Primary Health Care Unit located in the city of Fortaleza-CE. Results: Of the study participants, $74.2 \%$ were female, mean $61.8 \pm 11.9$ years old and within the age group of 54 to $69,77.1 \%$ race / brown color, $60.8 \%$ with incomplete fundamental level or complete and $70.6 \%$ with family income between 1 and 2 minimum wages. Of the evaluated hypertensive patients, $41.5 \%$ had Metabolic Syndrome and $76.5 \%$ had partial adherence to antihypertensive treatment. In the statistical evaluation of the association between the variables, it was verified that it did not exist $(p=0,149 \mathrm{e} p=0,642)$. The values regarding adherence to antihypertensive treatment were the same, both in patients with Metabolic Syndrome and in those who did not have this condition. Conclusions and implications for practice: High and partial antihypertensive therapy adherence was prevalent. The frequency of Metabolic Syndrome among hypertensive patients under study was high. Therefore, preventive measures for cardiovascular diseases and achievement of therapeutic goals must be implemented continuously. A challenge to be overcome by health professionals and services.

Keywords: Treatment Adherence and Compliance; Hypertension; Metabolic Syndrome; Measures of Association, Exposure, Risk or Outcome; Primary Health Care.

\section{RESUMEN}

Objetivo: Analizar la asociación entre la adherencia al tratamiento antihipertensivo y la aparición del síndrome metabólico en pacientes hipertensos en una unidad de atención primaria de salud. Método: Estudio analítico con corte transversal realizado con 306 pacientes hipertensos de una Unidad de Atención Primaria de Salud ubicada en la ciudad de Fortaleza-CE. Resultados: De los participantes en el estudio, el $74,2 \%$ eran mujeres, con una media de $61,8 \pm 11,9$ años y dentro del grupo de edad de 54 a $69,77,1 \%$ raza / color marrón, $60,8 \%$ con nivel fundamental incompleto o completo y $70.6 \%$ con ingresos familiares entre 1 y 2 salarios mínimos. De los pacientes hipertensos evaluados, el $41.5 \%$ tenía síndrome metabólico y el $76.5 \%$ tenía adherencia parcial al tratamiento antihipertensivo. En la evaluación estadística de la asociación entre las variables, se encontró que no existía $(p=0,149$ e $p=0,642)$. Los valores con respecto a la adherencia al tratamiento antihipertensivo fueron los mismos, tanto en pacientes con síndrome metabólico como en aquellos que no tenían esta condición. Conclusiones e implicaciones para la práctica: La adherencia a la terapia antihipertensiva alta y parcial fue prevalente. La frecuencia del síndrome metabólico entre los pacientes hipertensos en estudio fue elevada. Por lo tanto, las medidas preventivas para las enfermedades cardiovasculares y el logro de objetivos terapéuticos deben implementarse continuamente. Un desafío a superar por los profesionales y servicios de salud.

Palabras clave: Cumplimiento y Adherencia al Tratamiento; Hipertensión; Síndrome Metabólico; Medidas de Asociación, Exposición, Riesgo o Desenlace; Atención Primaria de Salud. 


\section{INTRODUÇÃO}

As doenças crônicas são consideradas um importante problema de saúde pública no panorama mundial, segundo a Organização Mundial da Saúde (OMS) ${ }^{1}$ estima-se que 13 milhões de pessoas morrem antes dos 70 anos, todos os anos, tendo como uma das principais causas as doenças cardiovasculares, seguidas de doença respiratória crônica, câncer e diabetes, sendo mais prevalentes nos países de baixa e baixa - média renda.

Dados da Secretaria de Vigilância em Saúde ${ }^{2}$ mostram que até o mês de setembro de 2018 houve um total de 178.362 casos de morte prematura, que corresponde à faixa etária de 30 a 69 anos, por Doenças Crônicas Não Transmissíveis (DCNT). No ano de 2017, este número foi maior, totalizando 225.431 até o mês de setembro do ano supracitado. Na região Nordeste estes dados

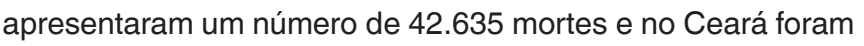
6.664 , sendo que no período decorrido entre o ano anterior até o mês de setembro, o número de mortes foi de 8.233.

Neste contexto, a Hipertensão Arterial Sistêmica (HAS) apresenta-se como uma das principais DCNT. A HAS, segundo a Sociedade Brasileira de Cardiologia, é uma condição clínica multifatorial caracterizada quando são registrados valores sistematicamente maiores ou iguais a $140 / 90 \mathrm{mmHg}$ no consultório. Essa condição também pode ser verificada diante de médias igualmente anormais $(\geq 130 / 80 \mathrm{mmHg}$ ) de 24 horas pela Monitorização Ambulatorial da Pressão Arterial (MAPA) ou pela Monitorização Residencial da Pressão Arterial (MRPA) $(\geq 135 / 85 \mathrm{mmHg}) .^{3}$

Atualmente, essa alteração fisiológica dos níveis pressóricos gera um dos mais importantes fatores de risco para o avanço de doenças sistêmicas no organismo humano, sendo responsável por pelo menos $40 \%$ das mortes por acidente vascular cerebral, $25 \%$ das mortes por doença arterial coronariana e, em combinação com o diabetes, por $50 \%$ dos casos de insuficiência renal terminal. ${ }^{4}$

Por isso, a HAS afeta de forma significativa e contínua a vida do paciente. Após o diagnóstico, são necessárias mudanças no estilo de vida e inclusão de tratamento medicamentoso para levar os valores da pressão arterial aos seus níveis normais e, assim, diminuir o risco cardiovascular. ${ }^{5}$

É de extrema importância que o profissional de saúde reconheça o nível de adesão terapêutico do paciente para que assim se possa intervir de acordo com a demanda apresentada, podendo reverter os casos de não adesão. Nesse processo de conscientização do paciente, é essencial que o profissional enfatize acerca da importância do controle pressórico para evitar a ocorrência de complicações cardiovasculares e melhorar a qualidade de vida do paciente.

A não cooperação e adesão ao tratamento anti-hipertensivo tem sido considerado um entrave para o controle pressórico. Essa falta de controle pressórico tem uma relação direta com a não adesão ao tratamento anti-hipertensivo. Portanto, a adesão terapêutica torna-se um componente essencial no tratamento da HAS e vem sendo conceituada como a convergência comportamental entre a prescrição do profissional de saúde e os hábitos comportamentais do paciente. ${ }^{6,7}$
Nesse cenário, a Síndrome Metabólica (SM) apresentase a partir de um conjunto de fatores de risco e tem como um dos desfechos desfavoráveis o desenvolvimento da doença cardiovascular. Estão associados à SM a circunferência abdominal (CA) elevada, o colesterol ligado à lipoproteína de alta densidade - high density lipoprotein (HDL-c) baixo, a pressão arterial sistêmica, os triglicerídeos e a glicemia elevados. ${ }^{8}$

Ressalta-se que esta coexistência pode sofrer alterações de acordo com a organização que a caracteriza. Diferentes organizações internacionais têm proposto definições da SM para adultos, utilizando às vezes os mesmos componentes, porém com pontos de corte diferentes na distribuição dos componentes empregados para defini-la.

A SM é a alteração metabólica mais comum e tem uma prevalência crescente em todo o mundo. ${ }^{9-11}$ No Brasil, essa condição, avaliada a partir da harmonização dos consensos internacionais e análise secundária da Pesquisa Nacional de Saúde de 2013, mostrou que 8,9\% (IC99\% 8,4-9,5) da população brasileira tem SM. ${ }^{12}$ Dados recentes de uma análise epidemiológica mostram que em população específica brasileira a prevalência de SM encontrada foi de $12,21 \%$. $^{13}$

A Sociedade Brasileira de Cardiologia infere que a SM se trata dessa associação de diversos fatores de risco cardiovasculares que associada, por sua vez, à HAS, sob a visão epidemiológica, é responsável pelo aumento em até 2,5 vezes da mortalidade por eventos cardiovasculares. Ressalta-se, ainda que, no caso de ocorrer essa associação, o tratamento, consiste na mudança no estilo de vida e na utilização ou não de medicamentos. Neste ponto, a utilização de medicamentos vai ser determinada se o nível da pressão arterial do indivíduo estiver igual ou superior a 140/90 mmHg. ${ }^{5}$

Estando a SM associada à HAS, condições que, quando associadas, aumentam o risco global para doenças cardiovasculares, torna-se necessário identificar se existem medidas de associação, exposição, risco ou desfecho entre adesão ao tratamento antihipertensivo e à ocorrência de síndrome metabólica e, assim, influenciar positivamente na saúde da população em geral.

Este estudo teve o objetivo de analisar a associação entre a adesão ao tratamento anti-hipertensivo e a ocorrência de síndrome metabólica em pacientes hipertensos de uma unidade de atenção primária à saúde.

\section{MÉTODO}

O presente estudo foi do tipo analítico com corte transversal e o levantamento de dados por meio da aplicação de questionário. Os dados foram coletados em uma instituição que integra a rede de Unidades de Atenção Primária à Saúde (UAPS) do município de Fortaleza, cuja abrangência envolve quatro bairros, sendo responsável por uma população estimada em cerca de 28.000 pessoas.

A população foi composta de adultos ( $\geq 18$ anos), de ambos os sexos, pertencentes à área de abrangência da UAPS e cadastrados nesta unidade de saúde como hipertensos. Foi realizado o cálculo para populações infinitas. ${ }^{14}$ Com base no resultado do cálculo, 
chegou-se a uma amostra de 282 participantes. Foram excluídos da amostra os pacientes agendados e que não compareceram à consulta. Foi acrescida uma margem de segurança de $8,5 \%$ de margem na amostra, totalizando 306 pacientes. Os pacientes foram convidados a participar da pesquisa no dia de seu atendimento de forma aleatória.

O nível de adesão ao tratamento anti-hipertensivo foi analisado a partir da aplicação do questionário Martín-Bayarre-Grau (MBG) desenvolvido em Cuba, ${ }^{15}$ o qual foi aplicado de forma individual na UAPS e respeitando a privacidade do participante por meio da sua aplicação em local reservado. Esse questionário é construído a partir da definição de adesão terapêutica, que é a ação ativa e voluntária do usuário de adotar um comportamento relacionado com o cumprimento do tratamento acertado de mútuo acordo com o seu médico. Este instrumento é autoaplicável, curto, de fácil aplicação e de convincentes possibilidades de resposta por parte do usuário. Possui 12 itens em forma de afirmações, com resposta tipo likert, com cinco possibilidades de resposta que vão de sempre a nunca. Para calcular a pontuação obtida por cada usuário, considera-se que a resposta nunca possui valor zero, quase nunca apresenta valor um, às vezes corresponde a dois, quase sempre se refere a três e para a resposta sempre é atribuído valor quatro, sendo 48 a totalidade dos pontos possíveis de alcançar. Os autores classificaram os valores, considerando as pontuações obtidas e consideram como aderidos totais os que obtêm 38 a 48 pontos; aderidos parciais, de 18 a 37 pontos, e não aderidos, de 0 a 17 pontos, de modo que se possa quantificar com rapidez e determinar três tipos de níveis de aderência: total, parcial e não aderido. O questionário apresentou uma consistência interna alfa de Crobach de 0,889 e a validação de conteúdo foi realizada por expert. ${ }^{16}$

Foi analisada também a prevalência da Síndrome Metabólica (SM) nesses pacientes com hipertensão, assim utilizou-se da definição do NCEP-ATP III, ${ }^{17}$ sendo necessário aferir os seguintes parâmetros dos pacientes: circunferência abdominal que foi aferida com fita métrica inelástica (Sanny® sn-4010, dois metros de comprimento, $0,5 \mathrm{~cm}$ de largura e resolução de $0,1 \mathrm{~mm}$ ) no ponto médio localizado entre a crista ilíaca anterior e o rebordo costal; medida da pressão arterial que foi realizada com aparelho digital modelo BM35 da marca Beurer $®$ com o intuito de minimizar a interferência do examinador no membro superior direito, seguindo o protocolo proposto pelas VII Diretrizes Brasileiras de Hipertensão Arterial; ${ }^{5}$ valores de triglicerídeos, o colesterol ligado à lipoproteína de alta densidade - high density lipoprotein (HDL-c) e glicemia em jejum foram obtidos por meio da consulta no prontuário eletrônico do paciente dos últimos exames laboratoriais realizados dentro de um período de até 1 ano.

Segundo a definição do NCEP-ATP III, é necessário que o paciente apresente no mínimo três dos critérios a seguir para caracterizá-lo com SM: obesidade abdominal por meio da medição da circunferência abdominal $>102 \mathrm{~cm}$ em homens e $>88 \mathrm{~cm}$ nas mulheres, triglicerídeos $\geq 150 \mathrm{mg} / \mathrm{dL}, \mathrm{HDL}-\mathrm{c}<40 \mathrm{mg} / \mathrm{dL}$ em homens e $<50 \mathrm{mg} / \mathrm{dL}$ nas mulheres, pressão arterial $\geq 130 \mathrm{mmHg}$ ou $\geq 85 \mathrm{mmHg}$ e Glicemia em jejum $\geq 110 \mathrm{mg} / \mathrm{dL}$. No caso de avaliação dos componentes glicemia, pressão arterial e triglicerídeos, o diagnóstico prévio de diabetes tipo 2, Hipertensão Arterial Sistêmica e o uso de hipolipemiantes, respectivamente, preenchem o critério diagnóstico. ${ }^{17}$

Após a coleta dos dados, evidenciou-se uma perda amostral em três dos critérios para a caracterização da prevalência da SM. Esse déficit de informação ocorreu nos fatores Triglicerídeos (40,8\%), HDL-c (40,5\%) e Glicemia de jejum (30,7\%) dos pacientes pesquisados. Tais parâmetros não inviabilizaram o estudo e suas análises, mas a falta dessas informações pode causar uma subestimação dos casos de SM entre os hipertensos. Ressalta-se a falta dessas informações no prontuário eletrônico do paciente, mesmo tendo sido realizado o exame alguns meses antes do período da coleta.

Os dados foram tabulados no software Microsoft Office Exce/® versão 2013 e, posteriormente, pelo software IBM $®$

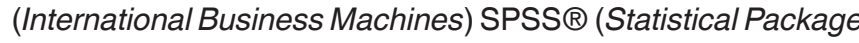
for the Social Sciences) versão 23, quando então os dados foram processados. Foram calculadas as medidas estatísticas descritivas médias e desvio-padrão das variáveis contínuas, assim como suas frequências absolutas e percentuais nas variáveis categóricas. As variáveis idade e renda familiar foram coletadas de maneira contínua e passaram por categorização, adotandose os critérios para a construção das classes (Amplitude Total, Intervalos de Classe pela fórmula de Sturges e tamanho de cada intervalo obtido pela divisão do valor da diferença entre o maior e o menor valor pelo número de intervalos de classe). ${ }^{14}$

Posteriormente, foi verificada a normalidade das variáveis através do Teste de Shapiro-Wilk e gráficos de dispersão, por fim, aplicou-se o Teste U de Mann-Whitney e Qui-quadrado de Independência para análise da adesão ao tratamento antihipertensivo com o diagnóstico de SM. Foi considerado em todos os testes o nível de significância estatística de $5 \%$.

O estudo respeitou as exigências formais contidas nas normas que envolvem seres humanos por meio da resolução 466/12 e foi aprovado pelo Comitê de Ética em Pesquisa da Universidade Estadual do Ceará com número de parecer 2.561.473, em março de 2018.

\section{RESULTADOS}

Os dados da Tabela 1 mostram que houve a predominância de mulheres (74,2\%). A média da idade dentre os pesquisados foi de 61,8 \pm 11,9 anos. Ao categorizar o dado contínuo em intervalos de classes para observar a distribuição da variável analisada, destacaram-se as faixas etárias de 62 a 69 anos $(27,5 \%)$ e de 54 a 61 anos (23,6\%), correspondendo a mais da metade dos pesquisados.

Em relação ao grau de escolaridade e renda familiar, 60,8\% dos pesquisados apresentavam nível fundamental incompleto ou completo e 70,6\% com renda familiar entre 1 e 2 salários mínimos.

Já sobre ao número de pessoas no domicílio e a raça/ cor auto referida, obteve-se $3,3 \pm 1,6$ pessoas e $77,1 \%$ se autodeclararam pardos. Na variável pessoas no domicílio, 
Tabela 1. Características sociodemográficas dos hipertensos pesquisados. Fortaleza, CE, Brasil. 2019. N=306.

\begin{tabular}{|c|c|c|c|c|}
\hline Características Sociodemográficas & №a & $\%^{\mathrm{b}}$ & Média & Desvio Padrão \\
\hline \multicolumn{5}{|l|}{ Sexo } \\
\hline Feminino & 227 & 74,2 & & \\
\hline Masculino & 79 & 25,8 & & \\
\hline Idade & & & 61,8 & 11,9 \\
\hline $22|-| 29$ & 1 & 0,3 & & \\
\hline $30|-| 37$ & 10 & 3,3 & & \\
\hline $38|-| 45$ & 15 & 4,9 & & \\
\hline $46|-| 53$ & 44 & 14,4 & & \\
\hline $54|-| 61$ & 72 & 23,6 & & \\
\hline $62|-| 69$ & 84 & 27,5 & & \\
\hline $70|-| 77$ & 55 & 18,1 & & \\
\hline $78|-| 85$ & 19 & 6,3 & & \\
\hline $86|-| 95$ & 5 & 1,6 & & \\
\hline \multicolumn{5}{|l|}{ Escolaridade } \\
\hline Analfabeto & 29 & 9,5 & & \\
\hline Nível fundamental incompleto/completo & 186 & 60,8 & & \\
\hline Nível médio incompleto/completo & 78 & 25,5 & & \\
\hline Nível superior incompleto/completo & 13 & 4,2 & & \\
\hline \multicolumn{5}{|l|}{ Renda familiar } \\
\hline$<1$ salário mínimo & 47 & 15,4 & & \\
\hline $1|-| 2$ salários mínimos & 216 & 70,6 & & \\
\hline $3|-| 4$ salários mínimos & 35 & 11,4 & & \\
\hline $5|-| 6$ salários mínimos & 6 & 2,0 & & \\
\hline$\geq 7$ salários mínimos & 2 & 0,6 & & \\
\hline Pessoas no domicílio & & & 3,3 & 1,6 \\
\hline 1 & 32 & 10,5 & & \\
\hline 2 & 72 & 23,5 & & \\
\hline 3 & 72 & 23,5 & & \\
\hline 4 & 70 & 22,9 & & \\
\hline 5 & 39 & 12,7 & & \\
\hline$\geq 6$ & 20 & 6,5 & & \\
\hline \multicolumn{5}{|l|}{ Raça/Cor } \\
\hline Amarela & 2 & 0,7 & & \\
\hline Branca & 59 & 19,3 & & \\
\hline Parda & 236 & 77,1 & & \\
\hline Preta & 9 & 2,9 & & \\
\hline
\end{tabular}

${ }^{a}$ Número; ${ }^{\text {bercentual; }}{ }^{\mathrm{N}} \mathrm{Na}$ variável idade teve a perda do registro de um dos pesquisados.

pôde-se descobrir que quase metade das famílias tinha de 2 a 3 pessoas no domicílio $(47,0 \%)$.

Com relação à ocupação dos pesquisados, destaca-se que mais da metade $(52,9 \%)$ tinha as seguintes ocupações: aposentados $(30,7 \%)$, do lar $(19,6 \%)$ ou pensionistas $(2,6 \%)$. Além disso, podemos constatar que $92,8 \%$ declararam não fumare $92,5 \%$ não faziam uso abusivo de álcool. Porém, mais da metade $(53,6 \%)$ era sedentário e tinha histórico de hipertensão arterial sistêmica na família $(85,9 \%)$.

Na Tabela2, pode-se ver as características clínicas avaliadas. Chama-se a atenção para o fato que os valores de Triglicerídeos, Colesterol ligado à lipoproteína de alta densidade - high density lipoprotein (HDL-c) e Glicemia de jejum não perfazem o total da amostra inicial do estudo, pois não houve a disponibilização dos 
Tabela 2. Características clínicas dos hipertensos pesquisados. Fortaleza, CE, Brasil. 2019. N=306.

\begin{tabular}{|c|c|c|c|c|c|}
\hline Características clínicas & №a & Mínimo & Máximo & Média & Desvio Padrão \\
\hline $\mathrm{CA}^{\mathrm{b}}(\mathrm{cm})$ - geral & 306 & 34,0 & 139,0 & 101,2 & 12,3 \\
\hline $\mathrm{CA}^{\mathrm{b}}(\mathrm{cm})$ - feminino & 227 & 34,0 & 139,0 & 100,5 & 12,9 \\
\hline $\mathrm{CA}^{\mathrm{b}}(\mathrm{cm})$ - masculino & 79 & 77,0 & 126,0 & 103,0 & 10,1 \\
\hline Triglicerídeos (mg/dL) & 181 & 45,0 & 761,0 & 172,6 & 100,0 \\
\hline $\mathrm{HDL}-\mathrm{c}^{c}(\mathrm{mg} / \mathrm{dL})$ - geral & 182 & 22,0 & 94,0 & 47,5 & 11,5 \\
\hline $\mathrm{HDL}-\mathrm{c}^{\mathrm{c}}(\mathrm{mg} / \mathrm{dL})$ - feminino & 135 & 28,0 & 84,0 & 48,2 & 11,0 \\
\hline $\mathrm{HDL}-\mathrm{c}^{\mathrm{c}}(\mathrm{mg} / \mathrm{dL})$ - masculino & 47 & 22,0 & 94,0 & 45,6 & 12,6 \\
\hline Glicemia em jejum (mg/dL) & 216 & 69,0 & 477,0 & 127,3 & 64,7 \\
\hline Pressão arterial sistêmica (mmHg) & 306 & 99 & 210 & 139,6 & 18,2 \\
\hline Pressão arterial diastólica $(\mathrm{mmHg})$ & 306 & 47 & 111 & 77,4 & 11,4 \\
\hline
\end{tabular}

${ }^{a}$ Número; ${ }^{b}$ Circunferência Abdominal; 'Colesterol ligado à lipoproteína de alta densidade - high density lipoprotein.

resultados no prontuário eletrônico do paciente, como descrito anteriormente.

O primeiro componente descrito foi a circunferência abdominal (CA). Os hipertensos apresentaram uma média geral de 101,2 $\pm 12,3 \mathrm{~cm}$ e um valor máximo de $139 \mathrm{~cm}$. Em relação ao sexo feminino, a média foi de $100,5 \pm 12,9 \mathrm{~cm}$ e ao sexo masculino, a média foi de $103,0 \pm 10,1 \mathrm{~cm}$.

Com relação aos triglicerídeos, a média deste item nos hipertensos foi de $172,6 \pm 100,0 \mathrm{mg} / \mathrm{dL}$, e um valor máximo de $761 \mathrm{mg} / \mathrm{dL}$. Já o componente HDL-c apresentou uma média geral para os hipertensos de 47,5 $\pm 11,5 \mathrm{mg} / \mathrm{dL}$. Esse componente, em referência ao sexo feminino, a média foi de 48,2 $\pm 11,0 \mathrm{mg} / \mathrm{dL}$ e ao sexo masculino, a média foi de $45,6 \pm 12,64 \mathrm{mg} / \mathrm{dL}$.

A glicemia em jejum apresentou uma média de 127,3 $\pm 64,7 \mathrm{mg} / \mathrm{dL}$ e valor máximo de $477 \mathrm{mg} / \mathrm{dL}$. As variações da PA foram: uma média de 139,6 \pm 18,2 mmHg na pressão arterial sistólica (PAS) e um valor máximo de $210 \mathrm{mmHg}$; a pressão arterial diastólica (PAD) apresentou uma média de 77,4 $\pm 11,4 \mathrm{mmHg}$ e valor máximo de $111 \mathrm{mmHg}$.

Posteriormente à referida descrição dos critérios para a caracterização da Síndrome Metabólica (SM), os hipertensos avaliados apresentaram uma prevalência de 41,5\% de SM de acordo com o NCEP-ATP III. Dentre os hipertensos, 35,3\% não dispunham de alguns dos exames laboratoriais que pudessem complementar a avaliação para verificar a ocorrência da SM como mostra os números da Tabela 2 , sendo assim foram classificados como "inconclusivos".

As respostas às 12 perguntas que compõem o Questionário Martín-Bayarre-Grau (MBG) que avalia a adesão ao tratamento anti-hipertensivo foram descritas a seguir.

Ao observar os dados apresentados na Tabela 3, pode-se constatar que o questionamento sobre a tomada de medicação no horário estabelecido e, de acordo com a dose indicada, foi de $41,5 \%$ e $70,9 \%$ dos hipertensos sempre tomam respectivamente; $31,4 \%$ afirmaram seguir, às vezes, as regras da dieta prescrita, próximo dos $35,6 \%$ que quase sempre seguiam e mais da metade (54,9\%) nunca realizam os exercícios físicos indicados. Os dois últimos itens descritos revelam uma certa resistência dos hipertensos do estudo na realização do tratamento não medicamentoso.

Referente ao comparecimento nas consultas marcadas, $83,3 \%$ afirmaram que sempre compareciam, porém apenas $35,0 \%$ responderam que sempre decidiam com o médico o tratamento a ser seguido. Condição corroborada por apenas $39,2 \%$ que responderam ter sempre a possibilidade de dar opinião no tratamento prescrito pelo médico e por $34,3 \%$ que responderam que sempre discutiam como cumprir o tratamento. Fatos que chamam atenção.

Ao exporem sobre acomodar os horários dos remédios nas atividades diárias, 59,8\% sempre o fizeram e 71,2\% nunca fizeram uso de lembretes para realização do tratamento. Somase isto ao fato de $57,5 \%$ darem incessantemente continuidade ao tratamento sem grandes esforços e de $64,1 \%$ terem sempre cumprido o tratamento sem supervisão de sua família ou amigos.

$\mathrm{Na}$ quantificação e posterior classificação da adesão ao tratamento anti-hipertensivo pelos dados obtidos da Tabela 3, obteve-se uma média de $31 \pm 7$ de pontuação nos questionários, chamando atenção, uma vez que a menor pontuação foi 5 . Este dado mostra que a classificação dos hipertensos, de maneira geral, é de aderência parcial, como recomendam os autores. Assim, pode-se verificar que a maioria dos pacientes $(76,5 \%)$ apresentava aderência parcial, 19,9\% apresentavam aderência total à terapêutica e apenas 3,6\% dos 306 hipertensos foram considerados não aderidos.

Por meio do teste de $U$ de Mann-Whitney mostrou-se que a adesão ao tratamento anti-hipertensivo não sofreu efeito em relação a ter ou não ter a Síndrome Metabólica $(U=3950,500$, $p=0,149$ ) quando analisados pelas pontuações brutas do questionário. Pois, de acordo com as hipóteses testadas, os dois grupos têm a mesma distribuição $\left(h_{0}\right)$ ou os dois grupos não têm a mesma distribuição $\left(h_{1}\right)$. Prevalecendo a hipótese nula. 
Tabela 3. Respostas dos hipertensos pesquisados ao questionário Martín-Bayarre-Grau (MBG). Fortaleza, CE, Brasil. 2019. N=306.

\begin{tabular}{|c|c|c|c|c|c|c|c|c|c|c|}
\hline \multirow{2}{*}{ Pergunta do MBG } & \multicolumn{2}{|c|}{ Sempre } & \multicolumn{2}{|c|}{ Quase sempre } & \multicolumn{2}{|c|}{ Às vezes } & \multicolumn{2}{|c|}{ Quase nunca } & \multicolumn{2}{|c|}{ Nunca } \\
\hline & №a & $\%^{\mathrm{b}}$ & №a & $\%^{\mathrm{b}}$ & №a & $\%^{\mathrm{b}}$ & №a & $\%^{\mathrm{b}}$ & №a & $\%^{\mathrm{b}}$ \\
\hline $\begin{array}{l}\text { Toma o medicamento nos } \\
\text { horários estabelecidos? }\end{array}$ & 127 & $41,5 \%$ & 101 & $33,0 \%$ & 56 & $18,3 \%$ & 18 & $5,9 \%$ & 4 & $1,3 \%$ \\
\hline $\begin{array}{l}\text { Você toma todas as doses } \\
\text { indicadas? }\end{array}$ & 217 & $70,9 \%$ & 47 & $15,4 \%$ & 24 & $7,8 \%$ & 13 & $4,2 \%$ & 5 & $1,6 \%$ \\
\hline Cumpre as regras da dieta? & 66 & $21,6 \%$ & 109 & $35,6 \%$ & 96 & $31,4 \%$ & 17 & $5,6 \%$ & 18 & $5,8 \%$ \\
\hline $\begin{array}{l}\text { Comparece às consultas } \\
\text { marcadas? }\end{array}$ & 255 & $83,3 \%$ & 31 & $10,1 \%$ & 12 & $3,9 \%$ & 4 & $1,3 \%$ & 4 & $1,3 \%$ \\
\hline $\begin{array}{l}\text { Realiza os exercícios físicos } \\
\text { indicados? }\end{array}$ & 58 & $19,0 \%$ & 25 & $8,2 \%$ & 33 & $10,8 \%$ & 22 & $7,2 \%$ & 168 & $54,9 \%$ \\
\hline $\begin{array}{l}\text { Acomoda os horários dos } \\
\text { remédios nas atividades diárias? }\end{array}$ & 183 & $59,8 \%$ & 57 & $18,6 \%$ & 36 & $11,8 \%$ & 16 & $5,2 \%$ & 14 & $5,6 \%$ \\
\hline $\begin{array}{l}\text { Você e seu médico decidem } \\
\text { juntos o tratamento a ser } \\
\text { seguido? }\end{array}$ & 107 & $35,0 \%$ & 43 & $14,1 \%$ & 26 & $8,5 \%$ & 39 & $12,7 \%$ & 91 & $29,7 \%$ \\
\hline $\begin{array}{l}\text { Cumpre o tratamento sem } \\
\text { supervisão de sua família ou } \\
\text { amigos? }\end{array}$ & 196 & $64,1 \%$ & 17 & $5,6 \%$ & 25 & $8,2 \%$ & 19 & $6,2 \%$ & 49 & $16,0 \%$ \\
\hline $\begin{array}{l}\text { Você dá continuidade ao } \\
\text { tratamento sem grandes } \\
\text { esforços? }\end{array}$ & 176 & $57,5 \%$ & 45 & $14,7 \%$ & 35 & $11,4 \%$ & 17 & $5,6 \%$ & 33 & $10,8 \%$ \\
\hline $\begin{array}{l}\text { Faz uso de lembretes para } \\
\text { realização do tratamento? }\end{array}$ & 43 & $14,1 \%$ & 7 & $2,3 \%$ & 17 & $5,6 \%$ & 21 & $6,9 \%$ & 218 & $71,2 \%$ \\
\hline $\begin{array}{l}\text { Você e seu médico discutem } \\
\text { como cumprir o tratamento? }\end{array}$ & 105 & $34,3 \%$ & 40 & $13,1 \%$ & 33 & $10,8 \%$ & 41 & $13,4 \%$ & 87 & $28,4 \%$ \\
\hline $\begin{array}{l}\text { Você tem a possibilidade de dar } \\
\text { a sua opinião no tratamento que } \\
\text { o seu médico prescreve? }\end{array}$ & 120 & $39,2 \%$ & 50 & $16,3 \%$ & 42 & $13,7 \%$ & 24 & $7,8 \%$ & 70 & $22,9 \%$ \\
\hline
\end{tabular}

anúmero; ${ }^{\text {PPercentual. }}$

A Tabela 4 mostra que 76,4\% apresentaram Síndrome Metabólica e aderência parcial ao tratamento anti-hipertensivo. Nos pesquisados que não tinham Síndrome Metabólica a aderência parcial também prevaleceu com 70,4\%.

O teste de Qui-quadrado de Independência sugeriu não existir alguma associação entre adesão ao tratamento anti-hipertensivo e diagnóstico de Síndrome Metabólica $\left[X^{2}(2)=0,888 ; p=0,642\right]$. Corroborado pela análise do grau de associação de variáveis categorias com o teste $\mathrm{V}$ de Cramer que indica apenas uma relação de 0,067 ou $6,7 \%$.

\section{DISCUSSÃO}

De acordo com o Ministério da Saúde ${ }^{18}$ os determinantes sociais de saúde são condições de vida inerentes ao ambiente, às características intrínsecas de cada indivíduo e às suas relações sociais que influenciam na saúde. Sendo assim, foi de extrema importância a avaliação dos dados sociodemográficos dos hipertensos mostrados na Tabela 1.

A partir da própria experiência profissional na Unidade de Atenção Primária à Saúde (UAPS), pode-se detectar que existem alguns fatores influenciadores na alta prevalência do sexo feminino entre os participantes, como por exemplo: a maior procura das mulheres pelos serviços de saúde. Raymundo e Pierin ${ }^{6}$ ressaltam que as mulheres, em relação à sua condição de saúde, parecem ter uma percepção mais acurada, tendo, assim, desenvolvido relação mais próxima com os serviços de saúde, isto é, decorrente também de seus atributos e funções reprodutivas.

Em estudo realizado na cidade de Fortaleza-CE, com 182 pacientes hipertensos cadastrados no Sistema de Gestão Clínica de Hipertensão Arterial e Diabetes Mellitus da atenção básica, obteve-se uma prevalência de $62,0 \%$ do total de hipertensos pertencentes ao sexo feminino, $66,9 \%$ idosos com idade média 
Tabela 4. Associação entre adesão ao tratamento anti-hipertensivo e a ocorrência de síndrome metabólica nos hipertensos pesquisados. Fortaleza, CE, Brasil. 2019. $\mathrm{N}=198$.

\begin{tabular}{|c|c|c|c|c|c|}
\hline \multirow{3}{*}{$\begin{array}{c}\text { Classificação do } \\
\text { Questionário MGB }\end{array}$} & \multicolumn{4}{|c|}{ Síndrome Metabólica } & \multirow{3}{*}{ p-valor } \\
\hline & \multicolumn{2}{|c|}{ Sim } & \multicolumn{2}{|c|}{ Não } & \\
\hline & №a & $\%^{b}$ & №a & $\%^{b}$ & \\
\hline Aderência total & 25 & $19,7 \%$ & 18 & $25,4 \%$ & \\
\hline Aderência parcial & 97 & $76,4 \%$ & 50 & $70,4 \%$ & $p=0,642^{c}$ \\
\hline Não aderido & 5 & $3,9 \%$ & 3 & $4,2 \%$ & \\
\hline Total & 127 & $100,0 \%$ & 71 & $100,0 \%$ & \\
\hline
\end{tabular}

${ }^{a}$ Número; ${ }^{b}$ Percentual; ${ }^{c} p$-valor para o teste qui-quadrado de Pearson.

de 64,5 anos. ${ }^{19}$ Dados que vão ao encontro dos achados dessa pesquisa e corroboram o levantamento de hipótese da não procura pelo atendimento em saúde por parte da população masculina, mesmo com a implantação de políticas de saúde voltada para esta população.

Consegue-se perceber que existe uma menor procura dos homens pelos serviços de saúde. Fato corroborado nas ações estratégicas da atenção básica no Sistema Único de Saúde. O panorama é voltado para a atenção à saúde da mulher em todo o seu ciclo de vida, dificultando o acompanhamento e detecção precoce das várias doenças. Mostra-se aqui o enfoque para a HAS e suas complicações. ${ }^{20}$

A ocupação "aposentado" é um fator que pode influenciar a adesão ao tratamento anti-hipertensivo, aumentando a dedicação ao tratamento pela disponibilidade, uma vez que as consultas ocorrem, geralmente, no período da manhã e podem coincidir com o horário de trabalho do paciente, assim os aposentados tendem a ter uma maior adesão. O que vai ao encontro do estudo de Lima et al. ${ }^{19}$ que apresenta 30,7\% de aposentados, $19,6 \%$ do Lar e $2,6 \%$ de pensionistas, totalizando $50,2 \%$ dos pesquisados.

Em um estudo realizado com pacientes hipertensos na cidade de São Paulo, 46,3\% não tinham ensino fundamental completo, ${ }^{20}$ corroborando o predomínio da baixa escolaridade entre os pacientes da pesquisa. Realidade que pode dificultar a adesão ao tratamento anti-hipertensivo pelo seu baixo letramento em saúde.

A renda é um fator que pode influenciar a adesão ao tratamento, uma vez que a maioria dos hipertensos do estudo $(70,6 \%)$ apresenta renda familiar entre 1 e 2 salários mínimos. Apesar de ser considerada uma renda "boa", dados da Pesquisa Nacional por Amostra de Domicílios Contínua (Pnadc) que trata de todas as fontes de rendimento, divulgada pelo Instituto Brasileiro de Geografia e Estatística (IBGE) mostraram que, no ano de 2018 , a renda média mensal de $60 \%$ dos trabalhadores brasileiros foi de $\mathrm{R} \$ 928$, valor inferior ao do salário mínimo em 2018 (R\$ 954). Esta situação se contrapõe aos dados apresentados, o que pode dificultar, muitas vezes, a obtenção de medicamentos que não estão disponíveis na rede pública ou para adquirir uma dieta mais saudável. ${ }^{21,22}$
O predomínio maior da declaração de não fumar e não fazer uso abusivo de álcool entre os hipertensos foi corroborado pelo estudo de Silva et al. ${ }^{23}$ mas, embora exista uma orientação de redução do consumo de álcool e tabaco na populações mais velhas, as intervenções específicas nestes fatores, pelos profissionais de saúde, devem ser contínuas a fim de detectar possíveis efeitos deletérios decorrentes dessa prática.

O sedentarismo em mais da metade dos pesquisados chama atenção, pois a relação entre o sedentarismo e a Síndrome Metabólica (SM), segundo a I Diretriz Brasileira Para o Diagnóstico e Tratamento da Síndrome Metabólica, ${ }^{24}$ aumenta em três a quatro vezes a prevalência da SM que tem essa condição.

A média dos valores de Pressão Arterial (PA) tende a ser alta no caso de não adesão ao tratamento anti-hipertensivo, pois a meta recomendada para os valores de PA de hipertensos com a terapêutica implementada é: $\mathrm{PA}<130 / 80 \mathrm{mmHg}$ e $<140 / 90 \mathrm{mmHg}$, de acordo com o estágio de hipertensão que o paciente apresenta, assim como o risco cardiovascular, ${ }^{5}$ o que foi constatado, como mostra a Tabela 2.

A não adesão ao tratamento anti-hipertensivo tem sido considerada um entrave para o controle pressórico. Esta falta de controle pressórico tem uma relação direta com a não adesão ao tratamento anti-hipertensivo. ${ }^{6}$ No estudo, pôde-se verificar que $76,5 \%$ dos pacientes apresentava aderência parcial, segundo a classificação do questionário Martín-Bayarre-Grau (MBG). Fato que mostra a importância da adesão terapêutica nos hipertensos. Este achado, somado aos dados da literatura mostram como a avaliação da adesão terapêutica torna-se um componente essencial ao tratamento da Hipertensão Arterial Sistêmica e vem sendo conceituada como a convergência comportamental entre a prescrição do profissional de saúde e os hábitos comportamentais do paciente, ${ }^{7}$ como pode ser observado pelos dados na Tabela 3.

A Tabela 4 exibe o cruzamento dos dados entre a adesão ao tratamento anti-hipertensivo e a SM, nos quais não foi possível mostrar, através da análise estatística, uma relação de associação. Ressalta-se que a não associação estatística entre estas variáveis, possivelmente, pode ser atribuída à alta pontuação do questionário MBG e sua distribuição equitativa entre quem tinha ou não o diagnóstico de SM. 


\section{CONCLUSÃO E IMPLICAÇÕES PARA A PRÁTICA}

Ao analisar os dados do estudo dos pacientes com hipertensão, foi possível concluir que existiu presença de aderência alta e parcial à terapêutica anti-hipertensiva prevalente, corroborando os dados da literatura mundial, sendo ainda este fato, um desafio para os serviços de saúde.

A Síndrome Metabólica também foi prevalente no estudo, mesmo sendo a condição mais comum que a compõe, embora não tenha sido encontrada alguma associação entre a sua presença e maior ou menor adesão terapêutica, ainda que os dados clínicos tenham colaborado para potenciais desfechos desfavoráveis associados a estas condições clínicas.

No entanto, é importante ressaltar que é necessário que se desenvolvam mais estudos envolvendo este tema, com a utilização de métodos diversos para que, assim, aumente-se a quantidade de conteúdo, com o intuito de auxiliar na melhoria da saúde da população e dissolver dúvidas ainda existentes. Além de cada vez mais serem implantadas medidas de prevenção para doenças cardiovasculares e alcance das metas terapêuticas.

\section{CONTRIBUIÇÕES DOS AUTORES}

Concepção e desenho da pesquisa: Geiciane Fonteles da Silva. Thereza Maria Magalhães Moreira.

Coleta de dados: Geiciane Fonteles da Silva.

Análise de dados: Paulo Sávio Fontenele Magalhães. Vagner Rodrigues Silva Junior.

Interpretação dos resultados: Paulo Sávio Fontenele Magalhães. Vagner Rodrigues Silva Junior. Thereza Maria Magalhães Moreira.

Redação e revisão crítica do manuscrito: Geiciane Fonteles da Silva. Paulo Sávio Fontenele Magalhães. Vagner Rodrigues Silva Junior. Thereza Maria Magalhães Moreira.

Aprovou a versão final do conteúdo publicado: Geiciane Fonteles da Silva. Paulo Sávio Fontenele Magalhães. Vagner Rodrigues Silva Junior. Thereza Maria Magalhães Moreira.

Responsabilidade por todos os aspectos da pesquisa e do manuscrito: Geiciane Fonteles da Silva. Paulo Sávio Fontenele Magalhães. Vagner Rodrigues Silva Junior. Thereza Maria Magalhães Moreira.

\section{EDITOR ASSOCIADO}

\author{
Gerson Luiz Marinho
}

\section{REFERÊNCIAS}

1. Organização Mundial da Saúde. World health statistics 2018: monitoring health for the SDGs, sustainable development goals [Internet]. Geneva: OMS; 2018 [citado 2020 jan 19]. Disponível em: https://apps.who.int/ iris/bitstream/handle/10665/272596/9789241565585-eng.pdf?ua=1

2. Ministério da Saúde (BR). Saúde Brasil 2018 uma análise de situação de saúde e das doenças e agravos crônicos: desafios e perspectivas [Internet]. Brasília: Ministério da Saúde; 2019 [citado 2020 jan 19]. Disponível em: http://bvsms.saude.gov.br/bvs/publicacoes/saude_ brasil_2018_analise_situacao_saude_doencas_agravos_cronicos_ desafios_perspectivas.pdf

3. Nobre F, Mion Jr D. Ambulatory blood pressure monitoring: five decades of more light and less shadows. Arq Bras Cardiol. 2016 jun;106(6):52837. http://dx.doi.org/10.5935/abc.20160065. PMid:27168473.

4. Monteiro AAF, Silva GCA, Silva LV, Cunha LS, Torres PA. Estudo sobre a adesão ao tratamento de hipertensão arterial sistêmica na UBSF de Três Poços. Braz. J. Hea. Rev. 2020;3(1):1289-305. http://dx.doi. org/10.34119/bjhrv3n1-099.

5. Malachias MVB, Póvoa RMS, Nogueira AR, Souza D, Costa LS, Magalhães ME. 7th Brazilian Guideline of Arterial Hypertension: Chapter 3 - clinical and complementary assessment. Arq Bras Cardiol. 2016;107(3 Supp 3):1-83. http://dx.doi.org/10.5935/abc.20160153.

6. Raymundo ACN, Pierin AMG. Adesão ao tratamento de hipertensos em um programa de gestão de doenças crônicas: estudo longitudinal retrospectivo. Rev Esc Enferm USP.2014 out;48(5):811-9. http://dx.doi. org/10.1590/S0080-6234201400005000006. PMid:25493484.

7. Borges JWP. Instrumento de avaliação da não adesão ao tratamento da hipertensão arterial: desenvolvimento e validação do conteúdo [dissertação]. Fortaleza (CE): Programa de Pós-Graduação em Cuidados Clínicos em Enfermagem e Saúde, Universidade Estadual do Ceará; 2012

8. Kuschnir MC, Bloch KV, Szklo M, Klein CH, Barufaldi LA, Abreu GA et al. ERICA: prevalence of metabolic syndrome in Brazilian adolescents. Rev Saude Publica. 2016;50(Suppl 1):11s. http://dx.doi.org/10.1590/ s01518-8787.2016050006701. PMid:26910546.

9. Diaz A, Espeche W, March C, Flores R, Parodi R, Genesio MA et al Prevalencia del síndrome metabólico en Argentina en los últimos 25 años: revisión sistemática de estudios observacionales poblacionales. Hipertens Riesgo Vasc. 2018 abr;35(2):64-9. http://dx.doi.org/10.1016/j. hipert.2017.08.003. PMid:28927871.

10. Zhang J, Liu Q, Long S, Guo C, Tan H. Prevalence of metabolic syndrome and its risk factors among 10.348 police officers in a large city of China: a cross - sectional study. Medicine (Baltimore). 2019;98(40):e17306. http://dx.doi.org/10.1097/MD.0000000000017306. PMid:31577722.

11. Rayyan Assi H, Ziv A, Dankner R. The metabolic syndrome and its components are differentially associated with chronic diseases in a high-risk population of 350000 adults: a cross-sectional study. Diabetes Metab Res Rev. 2019;35(4):e3121. http://dx.doi.org/10.1002/dmrr.3121. PMid:30600581.

12. Fortes MSR, Rosa SE, Coutinho W, Neves EB. Epidemiological study of metabolic syndrome in Brazilian soldiers. Arch Endocrinol Metab. 2019 ago;63(4):345-50. http://dx.doi.org/10.20945/2359-3997000000115. PMid:30916165.

13. Ramires EKNM, Menezes RCE, Longo-Silva G, Santos TG, Marinho PM, Silveira JAC. Prevalence and factors associated with metabolic syndrome among brazilian adult population: National Health Survey - 2013. Arq Bras Cardiol. 2018 maio;110(5):455-66. http://dx.doi. org/10.5935/abc.20180072. PMid:29898045.

14. Arango EDF. Bioestatística: teórica e computacional: com banco de dados reais em disco. $3^{a}$ ed. Rio de Janeiro: Guanabara Koogan;2012.

15. Libertad MA, Vea HDB, Ábalo JAG. Validación del cuestionario MBG (Martín-Bayarre-Grau) para evaluar la adherencia terapéutica en hipertensión arterial. Rev Cuba Salud Pública. [Internet]. 2008 mar; [citado 2019 mar 16];34(1). Disponível em: http://scielo.sld.cu/scielo. php?script=sci_arttext\&pid=S0864-34662008000100012\&lng=es

16. Rodrigues MTP. Adesão ao tratamento da hipertensão arterial sistêmica: desenvolvimento de um instrumento avaliativo com base na Teoria da Resposta ao Item (TRI) [tese]. Fortaleza (CE): Programa de PósGraduação em Saúde Coletiva, Universidade Estadual do Ceará; 2012.

17. National Cholesterol Education Program. Third report of the national cholesterol education program expert panel on detection, evaluation, and treatment of high blood cholesterol in adults (Adult Treatment Panel III). JAMA. 2011;285(19):2486-2497. http://dx.doi.org/10.1001/ jama.285.19.2486.

18. Ministério da Saúde (BR). Estratégias para o cuidado da pessoa com doença crônica: hipertensão arterial sistêmica [Internet]. Brasília (DF): Ministério da Saúde; 2014 [citado 2020 Mar 19]. Disponível em: http://bvsms.saude. gov.br/bvs/publicacoes/estrategias_cuidado_pessoa_doenca_cronica.pdf 
19. Lima DBS, Moreira TMM, Borges JWP, Rodrigues MTP. Association between treatment compliance and different types of cardiovascular complications in arterial hypertension patients. Texto Contexto Enferm. 2016;25(3):e0560015. http://dx.doi.org/10.1590/0104-07072016000560015.

20. Abreu RNDC. Adesão ao tratamento de pessoas com hipertensão arterial e complicações associadas: espaço para o cuidado clínico de enfermagem [dissertação]. Fortaleza (CE): Programa de Pós-graduação em Cuidados Clínicos em Enfermagem e Saúde, Universidade Estadual do Ceará;2007.

21. Figueiredo NN, Asakura L. Adesão ao tratamento anti-hipertensivo: dificuldades relacionadas a hipertensos. Acta Paul Enferm. 2010;23(6):7827. http://dx.doi.org/10.1590/S0103-21002010000600011.
22. Instituto Brasileiro de Geografia e Estatística. Projeções da população: Brasil e unidades da federação: revisão 2018 [Internet]. $2^{\mathrm{a}}$ ed. Rio de Janeiro: IBGE; 2018 [citado 2020 Abr 19]. Disponível em: https:// biblioteca.ibge.gov.br/visualizacao/livros/liv101597.pdf

23. Silva EB, Toniate VD, Tubaldini M, Ribeiro CHMA, Lo Prete AC. Perfi metabólico e terapêutica medicamentosa de pacientes com síndrome metabólica: um estudo retrospectivo. REAS/EJCH. 2020;12(3):e2689. http://dx.doi.org/10.25248/reas.e2689.2020.

24. Sociedade Brasileira de Hipertensao. I Diretriz Brasileira de Diagnóstico e Tratamento da Síndrome Metabólica. Arq. Bras. Cardiol.2005;84(Suppl 1):328. http://dx.doi.org/10.1590/S0066-782X2005000700001. 